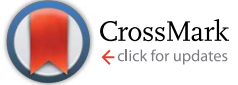

Cite this: RSC Adv., 2015, 5, 50938

Received 25th April 2015 Accepted 29th May 2015

DOI: 10.1039/c5ra07567j

www.rsc.org/advances

\section{Is a medium-range order pre-peak possible for ionic liquids without an aliphatic chain? $\uparrow$}

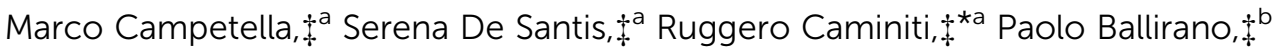

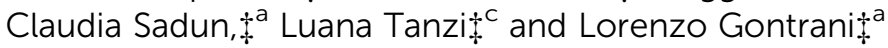

The combination of amino acids anions with a choline cation gives origin to a new and potentially important class of organic ionic liquids that might represent a viable and bio-compatible alternative with respect to the traditional ones. We present here a combined experimental and theoretical study of a choline-proline ionic liquid, using both large and small angle X-ray diffraction (WAXS-SAXS), and classical and $a b$ initio molecular dynamics calculations, in which we are able to point out for the first time the existence of a low $Q$ peak in the $X$-ray patterns in the absence of linear or branched alkyl chains. From the calculations, we can obtain theoretical scattering patterns that reproduce very nicely the experimental spectra in all $Q$ ranges, and from detailed analysis of the radial distribution functions (RDFs) and hydrogen bond patterns, we can state that very strong ion pairs are established in the liquid and the observed pre-peak can be ascribed to the interactions between atoms belonging to different ion pairs.

Ionic liquids (ILs) have been one of the most investigated research fields in material science in recent decades, owing to their outstanding chemical and technological properties. ${ }^{1-7}$ An interesting subset of ionic liquids prepared recently ${ }^{8}$ is composed of cations or anions derived from biomaterials obtainable from renewable sources, such as amino acids, ammonium, guanidinium and choline. More specifically, the combination of choline with amino-acid anions has been recently exploited to provide ionic liquids with very low toxicity to humans $\mathbf{s}^{\mathbf{1 , 5}, \mathbf{9}}$ and to the environment, though endowed of very desirable solvent properties, such as the ability to selectively dissolve lignin and cellulose from biomasses. ${ }^{10,11}$ Owing to the

\footnotetext{
${ }^{a}$ Department of Chemistry, University of Rome "La Sapienza", P.le A. Moro 5, Roma, Italy. E-mail: ruggero.caminiti@uniroma1.it; Fax: +3906490631

${ }^{b}$ Department of Earth Sciences, University of Rome "La Sapienza", P.le A. Moro 5, Roma, Italy

'Department of Physical and Chemical Sciences, University of L'Aquila, Via Vetoio (Coppito 1), 67100 Coppito, AQ, Italy

$\dagger$ Electronic supplementary information (ESI) available: Experimental procedures and computational details. See DOI: $10.1039 / \mathrm{c} 5 \mathrm{ra07567j}$

\$ All the authors contributed equally to this work.
}

relative novelty of these materials, the literature on their structural properties is still rather scarce and is limited to general theoretical studies of choline liquids ${ }^{\mathbf{1 2}}$ and to a recently developed force field, for which, though the systems examined contained aminoacids as the anions and a well-studied class of imidazolium compounds as the cations, ${ }^{\mathbf{1 3}}$ no experimental structural studies exist, to our knowledge. Among all the liquids of this class, we chose to report the results obtained for an interesting system synthesised in our lab (see ESI $\dagger$ ), cholineproline (see Fig. 1), since this combination exhibits an unexpected feature, namely the presence of a pre-peak or First Strong Diffraction Peak (FSDP) in the X-ray scattering patterns collected at small angles (Small Angle X-ray Scattering, SAXS), i.e. a peak falling at shorter $Q$ values than the principal peak of the liquid, analogous to what is generally observed for ionic liquids with medium-long alkyl chains. ${ }^{\mathbf{1 4 , 1 5}}$ The origin of this feature has been largely debated in several studies ${ }^{16,17}$ reaching the generally agreed hypothesis according to which the pre-peak is related to the existence of a medium-range order (MRO), mostly assignable to the 2nd and higher coordination shells (10-20 $\AA$ ) in the sample, ${ }^{\mathbf{1 8 , 1 9}}$ that in ionic liquids is reflected in the alternation of domains of different polarity. ${ }^{20,21}$ Such a mesoscopic separation tends to disappear when a polar group is introduced into the alkyl chain, ${ }^{17,22}$ or when the fragment can undergo some kind of intramolecular interaction that induces a partial folding of the chain. ${ }^{23}$ The latter phenomenon could be invoked for proline as well, since the four carbon atoms are arranged in a cycle, thus decreasing the available contact surface for hydrophobic interactions. The absence of well separated domains with cycloalkyl substitution was correlated to some thermodynamic data by our group ${ }^{24}$ and was described in a previous structure-activity relationship study by Tao et $a .^{25}$ Nevertheless, a clear pre-peak is visible in the small-angle X-ray scattering curve (Fig. 2), whose origin is therefore presumably different from the segregation of apolar domains within the charged matrix.

The first scattering peak of Fig. 2 falls at about $0.4 \AA^{-1}$ and is therefore contributed by medium-range structural correlations 


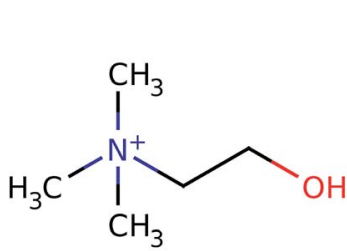

Choline

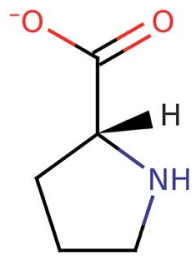

Proline
Fig. 1 Representation of the choline cation and proline anion.

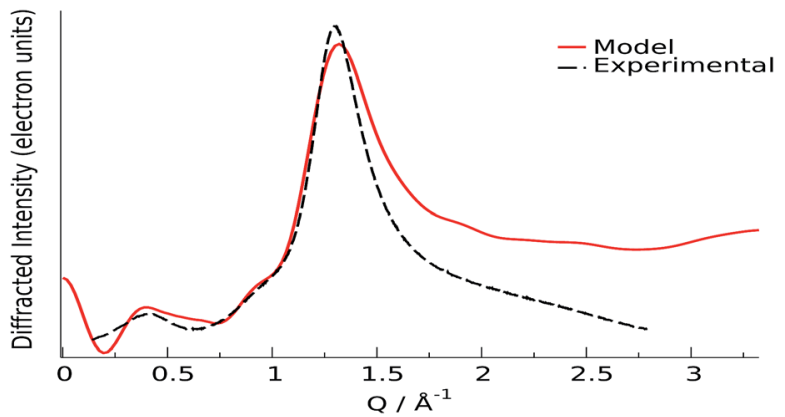

Fig. 2 Small angle $X$-ray scattering curve. Experimental: black dash; model: red line.
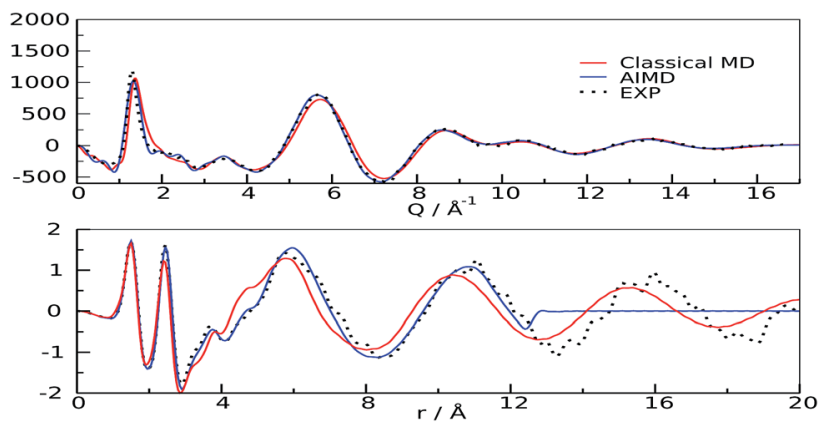

Fig. 3 Structure functions (upper panel) and radial distribution functions (lower panel). Experimental: black dots; classical MD: red; AIMD: blue

in the bulk liquid. ${ }^{18}$ The spatial dimension of a suitable interpretion model must therefore be twice as much as this value, owing to the minimum image convention of the Periodic Boundary Conditions (PBC) algorithm. ${ }^{26}$ Given the large dimension of the system (more than $10 \mathrm{~K}$ atoms) we decided to perform classical molecular dynamics simulations using the GPU-accelerated version of Amber software ${ }^{27}$ (see $\mathrm{ESI}^{\dagger}$ for details), with the two-body force field Gaff ${ }^{28}$ and properly tuned point charges. ${ }^{29}$ The agreement found using this model is very satisfactory for the low $Q$ range of Fig. 2, in particular regarding the position of the peaks. To further assess the model reliability, we confronted the theoretical structure function ${ }^{30-32}$ and the relative radial distribution function with the experimental analogues in the medium-long $Q$ range (Wide Angle X-ray Scattering, WAXS, i.e. short distances), and with an alternative higher accuracy theoretical model obtained from first-principle calculations (ab initio molecular dynamics). ${ }^{33}$ The scattering function reported in this $Q$-range (structure function $\mathrm{QIQM}(Q)$ ) is not suitable for highlighting the low- $Q$ peaks contributed by long distance correlations, but is generally preferred when radial patterns descriptive of the system are sought (see ESI, eqn $(2) \dagger)$. Very good agreements between the experimental and calculated patterns (structure functions and radial distribution functions) were found for all $Q$ and $r$ values, see Fig. 3, for both models, though the QM results are clearly superior in all spatial ranges, up to their allowed distance limit (half box length).

The quality of the agreement with the experimental data made us confident that our calculations were representative of the system, and could be used for further analysis of the microscopic structure of the liquid. One of the most important rheological features of choline-aminoacid liquids is the very high viscosity they possess, that can be ascribed to the existence of very strong interactions between the ions. Among all the possible forms of interactions, hydrogen bonds play a major role, as already pointed out in the study by Benedetto et $a l .{ }^{12} \mathrm{In}$ fact, the relative radial distribution function (between Cho and Pro oxygen atoms), reported in Fig. 4-panel A, shows a sharp peak whose maximum value $(2.65 \AA$ and $2.62 \AA$, for classical and QM models, respectively), falls at significantly shorter distance than the equilibrium value of $2.75-2.8 \AA$ of liquid water ${ }^{34,35}$ This observation suggests that cations and anions give origin to strong ion pairs. To better characterize the cation-anion interaction we have calculated the relative Combined Distribution Function $(\mathrm{CDF}){ }^{36}{ }^{36}$ which correlates the distance between the cation and anion oxygen atoms and the distribution of the angle formed by two vectors, the former corresponding to the $\mathrm{O}-\mathrm{H}$ bond and the latter connecting the two hetero atoms; the relative function is shown in Fig. 4-panel B. The region of maximum occurrence in the contour plot corresponds to the distance range from 2.5 to $2.9 \AA$ and to the angular interval of $160-180$ degrees; these distance and angle values were used as threshold criteria to classify the $\mathrm{O}-\mathrm{H} \cdots \mathrm{O}$ contacts as hydrogen bonds. ${ }^{37-39}$

The occurrence and time duration of these hydrogen bonds were derived from a detailed analysis of the AIMD trajectory (see ESI, computational details $\dagger$ ), whose shorter time step and more correct description of the interaction potential was considered more appropriate for investigating the $\mathrm{H}$-bond spatial features and appraising its time evolution. Over a total of 50 ion pairs, we found that 38 cations and 30 anions form persistent bonds during the observed time (40 ps); such cations and anions remain bonded for 35.9 and 38.7 ps, respectively. The lower number of anions involved is compliant with the presence of two H-bond sites in the carboxylate. To quantify such an interaction, we performed an Umbrella Sampling (U. S.) calculation $^{40}$ (ESI, computational details $\uparrow$ ) on the QM trajectory using the $\mathrm{O}(\mathrm{Cho}) \cdots \mathrm{O}(\mathrm{Pro})$ distance as the reaction coordinate; the resulting potential of the mean force, ${ }^{41}$ shown in Fig. 4-panel C, has a minimum of about $7 \mathrm{kcal} \mathrm{mol}^{-1}$ located at $2.62 \AA$. Such a value is similar to the values reported for the carboxylic acid dimers in ref. $42-44$ and is stronger than for liquid water (about $5 \mathrm{kcal} \mathrm{mol}^{-1}$ ), in compliance with the shorter $\mathrm{O} \cdots \mathrm{O}$ distance observed in the radial distribution function. 


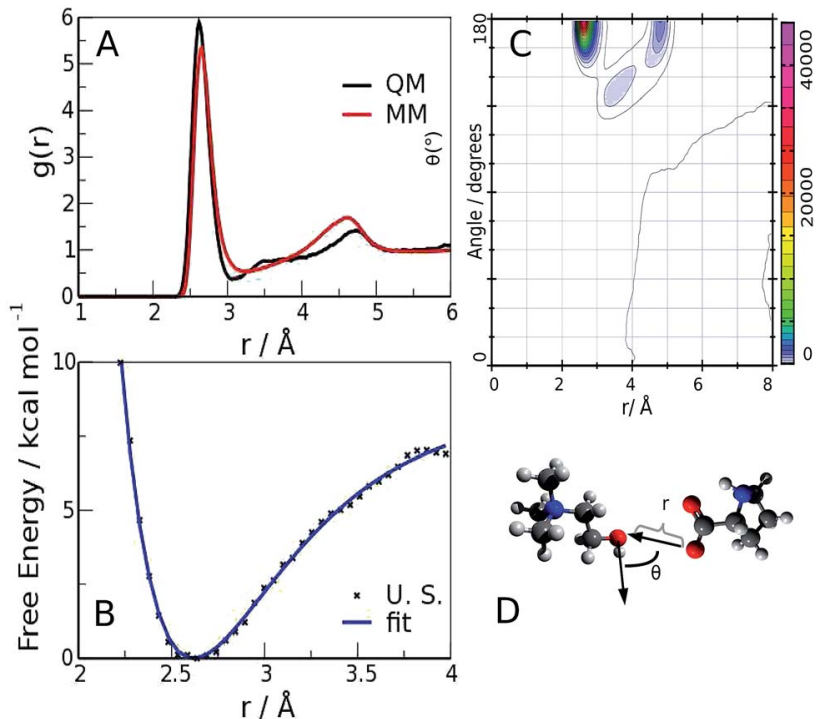

Fig. $4 \mathrm{O}$ (Cho) $\cdots O$ (Pro) radial distribution function (panel A) combined radial-angular distribution function (panel B), free energy profile of the $\mathrm{H}$-bond interaction (panel $\mathrm{C}$ ) and sketch of the $\mathrm{H}$-bond (panel D). The blue line is a fit of the free energy data with a Morse-type function.

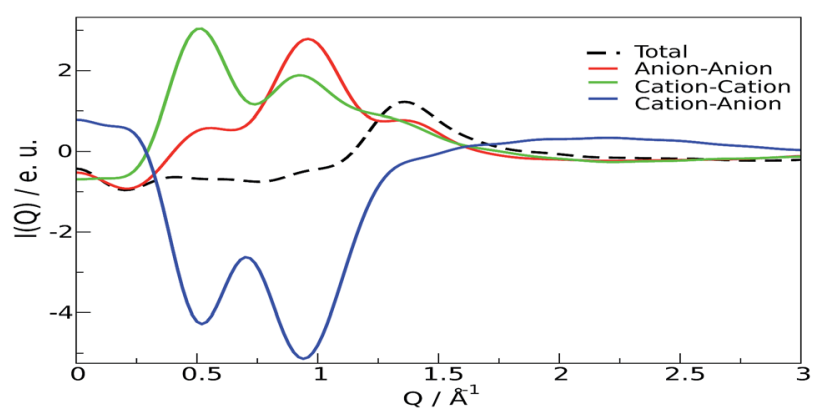

Fig. 5 Individual contributions to the low $Q$ diffraction pattern. Black: total; red: cation-cation; green: anion-anion; blue: cation-anion.

From these results, it can be stated that this ion pair interaction is largely responsible for the strong structural ordering at low distances; the "structure" of the liquid stems from the packing of the fundamental units, the ion pairs. The overall disposition of the ions, through the interference of their scattered beams, gives origin to the observed spectra Fig. 3 and 4. The total scattering pattern can be divided into the individual contributions coming from each pair of scatterers (see ESI, eqn (3) $\dagger$ ), which can be, in turn, clustered into group terms, like anion and cation. This analysis is shown in Fig. 5, where the whole pattern (black) is decomposed into anion-anion, cation-cation and cation-anion contributions. It can be seen that homologous correlations (cation-cation and anion-anion) lead to the positive peaks at around $0.5 \AA^{-1}$ and are counterbalanced by the intense negative trough due to the mixed cation-anion terms. The resultant curve shows the presence of a pre-peak located around $0.4 \AA^{-1}$ that is also observed in the experimental data. The structural correlations underlying the two peaks can be related to an average
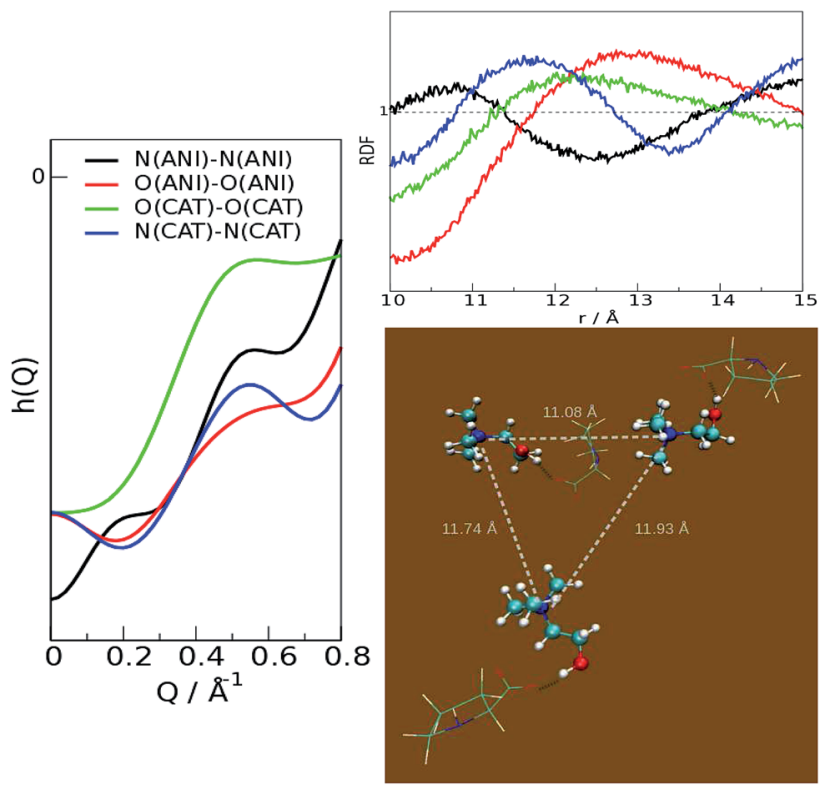

Fig. 6 Partial contributions of the heteroatoms. Left: scattering function $H(Q)$; right, top: radial distribution functions; right, bottom: typical second-shell arrangement of the ion pairs.

"effective distance" of about $2 \pi / Q \approx 12.5 \AA$, that can be easily appreciated in the $g(r)^{\prime}$ 's between the nitrogen and oxygen atoms of the cations and anions, respectively, and in their corresponding Fourier-transform $H(Q)$, the partial contributions to the scattering function (see ESI, eqn (3) $\dagger$ ) that show a consensus peak at $0.5 \AA^{-1}$. All the curves are reported in Fig. 6, together with the typical geometrical arrangement of the ions taken from a trajectory frame, in which the cation-cation contacts are highlighted.

Summarizing, the results shown indicate that in the cholineproline ionic liquid the most important structural feature is the existence of strong ion pairs that mutually solvate one another. The diffraction pre-peak observed can be ascribed to the scattering of second-neighbour groups. In future work, we plan to investigate if this motif can be recognized in other systems lacking hydrophobic moieties, e.g. aromatic rings.

\section{Acknowledgements}

We are deeply grateful to Enrico Bodo, for his support and for the very helpful discussions. We also thank the Sapienza University of Rome for financial support through grant C26H14P9R2, Prof. Ruggero Caminiti (Sapienza University of Rome Chemistry Department) for providing free usage of the Narten computing cluster facility and the Sapienza Nanotechnology Center (CNIS) for providing access to the D8 Diffractometer. Computational support from PRACE (grant no. 2013091962) is also acknowledged.

\section{References}

1 P. Nockemann, B. Thijs, K. Driesen, C. R. Janssen, K. V. Hecke, L. V. Meervelt, S. Kossmann, B. Kirchner and K. Binnemans, J. Phys. Chem. B, 2007, 111, 5254-5263. 
2 Y. Fukaya, Y. Iizuka, K. Sekikawa and H. Ohno, Green Chem., 2007, 9, 1155-1157.

3 Y. Yu, X. Lu, Q. Zhou, K. Dong, H. Yao and S. Zhang, Chem.Eur. J., 2008, 14, 11174-11182.

4 J.-C. Plaquevent, J. Levillain, F. Guillen, C. Malhiac and A.-C. Gaumont, Chem. Rev., 2008, 108, 5035-5060.

5 M. Petkovic, J. L. Ferguson, H. Q. N. Gunaratne, R. Ferreira, M. C. Leitao, K. R. Seddon, L. P. N. Rebelo and C. S. Pereira, Green Chem., 2010, 12, 643-649.

6 A. J. L. Costa, M. R. C. Soromenho, K. Shimizu, I. M. Marrucho, J. M. S. S. Esperança, J. N. C. Lopes and L. P. N. Rebelo, ChemPhysChem, 2012, 13, 1902-1909.

7 Y. Gao, S. W. Arritt, B. Twamley and J. M. Shreeve, Inorg. Chem., 2005, 44, 1704-1712.

8 C. Rüss and B. Konig, Green Chem., 2012, 14, 2969-2982.

9 K. D. Weaver, H. J. Kim, J. Sun, D. R. MacFarlane and G. D. Elliott, Green Chem., 2010, 12, 507-513.

10 Q.-P. Liu, X.-D. Hou, N. Li and M.-H. Zong, Green Chem., 2012, 14, 304-307.

11 X.-D. Hou, T. J. Smith, N. Li and M.-H. Zong, Biotechnol. Bioeng., 2012, 109, 2484-2493.

12 A. Benedetto, E. Bodo, L. Gontrani, P. Ballone and R. Caminiti, J. Phys. Chem. B, 2014, 118, 2471-2486.

13 V. V. Chaban and E. E. Fileti, J. Phys. Chem. B, 2015, 119, 3824-3828.

14 O. Russina, A. Triolo, L. Gontrani and R. Caminiti, J. Phys. Chem. Lett., 2012, 3, 27-33.

15 M. Campetella, L. Gontrani, F. Leonelli, L. Bencivenni and R. Caminiti, ChemPhysChem, 2015, 16, 197-203.

16 C. Hardacre, J. D. Holbrey, C. L. Mullan, T. G. A. Youngs and D. T. Bowron, J. Chem. Phys., 2010, 133, 074510.

17 O. Russina, A. Triolo, L. Gontrani, R. Caminiti, D. Xiao, L. G. Hines Jr, R. A. Bartsch, E. L. Quitevis, N. Plechkova and K. R. Seddon, J. Phys.: Condens. Matter, 2009, 21, 424121.

18 P. H. Gaskell and D. J. Wallis, Phys. Rev. Lett., 1996, 76, 6669.

19 G. Lucovsky and J. C. Phillips, Phys. Status Solidi B, 2009, 246, 1806-1812.

20 H. K. Kashyap, C. S. Santos, R. P. Daly, J. J. Hettige, N. S. Murthy, H. Shirota, E. W. Castner and C. J. Margulis, J. Phys. Chem. B, 2013, 117, 1130-1135.

21 A. Triolo, O. Russina, H.-J. Bleif and E. Di Cola, J. Phys. Chem. $B, 2007,111,4641-4644$.

22 A. Triolo, O. Russina, R. Caminiti, H. Shirota, H. Y. Lee, C. S. Santos, N. S. Murthy and E. W. Castner Jr, Chem. Commun., 2012, 48, 4959-4961.
23 M. Campetella, L. Gontrani, E. Bodo, F. Ceccacci, F. C. Marincola and R. Caminiti, J. Chem. Phys., 2013, 138, 184506.

24 S. De Santis, G. Masci, F. Casciotta, R. Caminiti, E. Scarpellini, M. Campetella and L. Gontrani, Phys. Chem. Chem. Phys., 2015, in revision.

25 D.-J. Tao, Z. Cheng, F.-F. Chen, Z.-M. Li, N. Hu and X.-S. Chen, J. Chem. Eng. Data, 2013, 58, 1542-1548.

26 M. P. Allen and D. J. Tildesley, Computer Simulation of Liquids, Oxford University Press, 1987.

27 A. W. Goetz, M. J. Williamson, X. Dong, P. Duncan, S. Le Grand and R. C. Walker, J. Chem. Theory Comput., 2012, 8, 1542-1555.

28 J. Wang, W. Wang, P. A. Kollman and D. A. Case, J. Mol. Graphics Modell., 2006, 25, 247-260.

29 Y. Zhang and E. J. Maginn, J. Phys. Chem. B, 2012, 116, 10036-10048.

30 L. Gontrani, O. Russina, F. C. Marincola and R. Caminiti, J. Chem. Phys., 2009, 131, 244503.

31 V. R. Albertini, L. Bencivenni, R. Caminiti, F. Cilloco and C. Sadun, J. Macromol. Sci., Part B: Phys., 1996, 35, 199-213.

32 The structure of Ionic Liquids, ed. R. Caminiti and L. Gontrani, Springer, 2014, vol. 193.

33 J. Hutter, M. Iannuzzi, F. Schiffmann and J. VandeVondele, WIREs Comput. Mol. Sci., 2014, 4, 15-25.

34 T. Head-Gordon and G. Hura, Chem. Rev., 2002, 102, 26512670.

35 A. K. Soper, ISRN Phys. Chem., 2013, 2013, 67.

36 M. Brehm and B. Kirchner, J. Chem. Inf. Model., 2011, 51, 2007-2023.

37 K. Dong and S. Zhang, Chem.-Eur. J., 2012, 18, 2748-2761.

38 E. Bodo, A. Sferrazza, R. Caminiti, S. Mangialardo and P. Postorino, J. Chem. Phys., 2013, 139, 144309.

39 L. Gontrani, E. Bodo, A. Triolo, F. Leonelli, P. D'angelo, V. Migliorati and R. Caminiti, J. Phys. Chem. B, 2012, 116, 13024-13032.

40 G. Torrie and J. Valleau, J. Comput. Phys., 1977, 23, 187-199. 41 S. Kumar, J. M. Rosenberg, D. Bouzida, R. H. Swendsen and P. A. Kollman, J. Comput. Chem., 1995, 16, 1339-1350.

42 T. Neuheuser, B. A. Hess, C. Reutel and E. Weber, J. Phys. Chem., 1994, 98, 6459-6467.

43 Y. Gu, T. Kar and S. Scheiner, J. Am. Chem. Soc., 1999, 121, 9411-9422.

44 M. W. Feyereisen, D. Feller and D. A. Dixon, J. Phys. Chem., 1996, 100, 2993-2997. 